\title{
Dipolar Bose-Einstein condensates with large scattering length
}

\author{
Luis E. Young-S,,$^{1, *}$ S. K. Adhikari, ${ }^{1, \dagger}$ and P. Muruganandam ${ }^{1,2, \ddagger}$ \\ ${ }^{1}$ Instituto de Física Teórica, UNESP-Universidade Estadual Paulista, 01.140-070 São Paulo, São Paulo, Brazil \\ ${ }^{2}$ School of Physics, Bharathidasan University, Palkalaiperur Campus, Tiruchirappalli 620024, Tamilnadu, India \\ (Received 29 December 2011; revised manuscript received 30 January 2012; published 14 March 2012)
}

\begin{abstract}
A uniform dilute Bose gas of known density has a universal behavior as the atomic scattering length tends to infinity at unitarity while most of its properties are determined by a universal parameter $\xi$ relating the energies of the noninteracting and unitary gases. The usual mean-field equation is not valid in this limit, and beyond mean-field corrections become important. We use a dynamical model including such corrections to investigate a trapped disk-shaped dipolar Bose-Einstein condensate (BEC) and a dipolar BEC vortex for large scattering length. We study the sensitivity of our results on the parameter $\xi$ and discuss the possibility of extracting the value of this parameter from experimental observables.
\end{abstract}

DOI: 10.1103/PhysRevA.85.033619

PACS number(s): 03.75.Hh, 03.75.Kk, 05.30.Jp

\section{INTRODUCTION}

The properties of a uniform dilute interacting (Bose or Fermi) atomic quantum gas interacting with an $S$-wave contact interaction at zero temperature is determined by two scales: the atomic scattering length $a$ and density $\bar{n}$. As $a \rightarrow \infty$ at unitarity, the first scale is not of concern, and the observables of the gas are determined solely by density and the gas exhibits a universal behavior. Bulk chemical potential $\bar{\mu}$ of the unitary gas is proportional to the only available energy scale, the Fermi energy (or the chemical potential of the noninteracting gas) $E_{F}=\hbar^{2}\left(6 \pi^{2} \bar{n}\right)^{2 / 3} / 2 m$, so that $\bar{\mu}=\xi E_{F}$, where $\xi$ is a universal parameter and $m$ is the mass of an atom [1-16]. Similarly, the energy per particle $\bar{E}$ of the unitary gas is proportional to the energy per particle $E\left(\equiv 3 E_{F} / 5\right)$ of the noninteracting gas: $\bar{E}=\xi E[3,4]$. The Fermi energy is a physically meaningful quantity for the Fermi gas, but the same can also be used as an energy scale for the Bose gas $[2,12,14]$. The Bose and Fermi gases behave similarly at unitarity because the Bose gas exhibits fermionization. If this fermionization of the Bose gas is absolute, then $\xi$ should be the same for the Bose and Fermi gases.

The parameter $\xi$ relating the energy of the noninteracting and unitary gases has been "measured" experimentally from a study at or near unitarity of the density [1,5-7], or of groundstate energy, or of sound velocity [8] of a trapped Fermi gas. But a similar experiment is more difficult for a Bose-Einstein condensate (BEC) due to a large probability of three-body loss by molecule formation at or near unitarity, which is the threshold for molecule formation [12]. In the weak-coupling limit $\left(a n^{1 / 3}<1\right)$ the mean-field Gross-Pitaevskii (GP) equation gives a good description of a trapped BEC, where $n$ is the density. In the strong-coupling regime $\left(a n^{1 / 3}>1\right)$ the GP equation highly overestimates the atomic contact interaction and leads to unphysical results. Experimental activities to access the strong-coupling regime of a BEC, to test the beyond mean-field corrections [17,18], and to extract the parameter $\xi$ from these studies have just began [12]. Although

\footnotetext{
*lyoung@ift.unesp.br

†adhikari44@yahoo.com

† anand@cnld.bdu.ac.in
}

unitarity is also the threshold for molecule formation in a two-component (spin up and down) Fermi gas, the probability of formation of diatomic molecules is highly suppressed in this case due to Pauli repulsion among spin-parallel fermions in the three-fermion system and hence is not of concern [1].

Lately, BECs of ${ }^{52} \mathrm{Cr}[19,20]$ and ${ }^{164} \mathrm{Dy}[21,22]$ with large dipolar interaction have been observed and studied. The interatomic interaction now has two components: an $S$-wave contact interaction and an anisotropic long-range dipolar interaction. This allows one to study the dipolar BEC with a variable contact interaction $[19,22]$ using a Feshbach resonance [23]. The intrinsically anisotropic dipolar BEC [24] has many distinct features $[19,20,25-28]$. The stability of a dipolar BEC depends not only on the scattering length, but also on the trap geometry $[19,25,27]$. A disk-shaped trap leads to a repulsive dipolar interaction and the dipolar BEC is more stable, whereas a cigar-shaped trap yields an attractive dipolar interaction and hence favors a collapse instability [19,27,29].

We study the static and dynamic properties of a disk-shaped dipolar BEC and dipolar BEC vortex, with the dipole moments aligned perpendicular to the plane of the disk, for large scattering length using a beyond-mean-field model $[16,30]$ for the BEC-unitarity crossover. In this paper we consider the strong-coupling limit of the contact interaction only and not the same limit of dipolar interaction. In the weak-coupling limit, this crossover model reduces to the GP equation and the Lee-Huang-Yang (LHY) correction [31], whereas in the strong-coupling regime of large scattering length it reduces to the universal result at unitarity. We find that the radial densities are sensitive to the parameter $\xi$ in the strong-coupling regime, and hence a study of density is expected to yield information about this parameter. However, the frequency of oscillation of the dipolar BEC is found to be insensitive to this parameter. From a study of vortices in a disk-shaped dipolar BEC we find that both density and radius of vortex core are sensitive to this parameter in the strong-coupling regime.

In the disk configuration, the dipolar interaction is highly repulsive, as parallel dipoles arranged in a plane with the dipole moment perpendicular to the plane repel each other $[19,20]$. The strongly repulsive dipolar interaction should reduce three-body loss by molecule formation in the strong-coupling regime, as the rate of the reaction $3 A \rightarrow A_{2}+A$ should be 
suppressed in this setting with $A$ representing a dipolar atom and $A_{2}$ a molecule. Also, as both the contact and long-range dipolar interactions contribute to molecule formation, the threshold for molecule formation will be displaced from unitarity, specially in strongly dipolar BECs, thus further reducing three-body loss by molecule formation and creating a new scenario of experiment with a dipolar BEC in the strong-coupling regime to determine the parameter $\xi$.

In Sec. II we present the mean-field and beyond-mean-field models to study a dipolar BEC in the weak- and strongcoupling regimes as well as along the BEC-unitarity crossover as the scattering length $a$ is increased. We also present a Gaussian variational formulation for its solution at unitarity. In Sec. III we present the results of numerical and variational studies of density, root-mean square (rms) sizes, chemical potential, and frequencies of radial and axial oscillations of a disk-shaped dipolar BEC and BEC vortex. Finally in Sec. IV we present a brief summary and conclusion.

\section{ANALYTICAL CONSIDERATION}

\section{A. Dipolar Gross-Pitaevskii equation}

We consider a disk-shaped dipolar BEC of $N$ atoms, each of mass $m$, using the GP equation [19]

$$
i \frac{\partial \phi(\mathbf{r}, t)}{\partial t}=\left[-\frac{\nabla^{2}}{2}+V+\bar{\mu}(a, N)+F\left(a_{d d}, N\right)\right] \phi(\mathbf{r}, t),
$$

with the bulk chemical potential

$$
\bar{\mu}(a, N)=4 \pi a n
$$

and density $n=N|\phi|^{2}$. Here the dipolar nonlinearity $F\left(a_{d d}, N\right)=N \int U_{d d}\left(\mathbf{r}-\mathbf{r}^{\prime}\right)\left|\phi\left(\mathbf{r}^{\prime}, t\right)\right|^{2} d \mathbf{r}^{\prime}$,

$$
V=\frac{1}{2}\left(x^{2}+y^{2}+\lambda^{2} z^{2}\right)
$$

is the harmonic trap, $\mathbf{r} \equiv\{x, y, z\} \equiv\{\rho, z\}, \quad U_{d d}(\mathbf{R})=$ $3 a_{d d}\left(1-3 \cos ^{2} \theta\right) / R^{3}, \mathbf{R}=\mathbf{r}-\mathbf{r}^{\prime}$, normalization $\int \phi(\mathbf{r})^{2} d \mathbf{r}=$ $1, \theta$ the angle between $\mathbf{R}$ and the polarization direction $z$, $\lambda \gg 1$ the trap anisotropy, $a_{d d}=\mu_{0} \tilde{\mu}^{2} m /\left(12 \pi \hbar^{2}\right)$ the strength of dipolar interaction, $\mu_{0}$ the permeability of free space, and $\tilde{\mu}$ the (magnetic) dipole moment. In Eq. (1) the $\mathbf{r}$ and $t$ dependence of $\bar{\mu}$ and $F$ are not explicitly shown, and length is measured in units of $l_{0} \equiv \sqrt{\hbar / m \omega}$, where $\omega$ is the harmonic trap frequency in $x$ or $y$ directions, and time $t$ in units of $t_{0}=\omega^{-1}$. At unitarity, the bulk chemical potential of Eq. (1) is independent of $a$ and is [2]

$$
\bar{\mu}(a, N)=\frac{1}{2} \xi\left(6 \pi^{2} n\right)^{2 / 3} .
$$

To obtain a quantized vortex of unit angular momentum $\hbar$ around the $z$ axis, we introduce a phase (equal to the azimuthal angle) in the wave function [32]. This procedure introduces a centrifugal term $1 /\left[2\left(x^{2}+y^{2}\right)\right]$ in the potential of the GP equation so that

$$
V=\frac{1}{2}\left(x^{2}+y^{2}+\lambda^{2} z^{2}\right)+\frac{1}{2\left(x^{2}+y^{2}\right)} .
$$

We adopt this procedure to study an axially symmetric vortex in a disk-shaped dipolar BEC.

\section{B. BEC-unitarity crossover}

Lee, Huang, and Yang (LHY) [31] obtained the leading terms of the beyond-mean-field expression for energy of a uniform Bose gas from which the following expression for the bulk chemical potential can be obtained [33]:

$$
\bar{\mu}(a, N)=4 \pi \operatorname{an}\left[1+\alpha a^{3 / 2} \sqrt{n}\right] .
$$

The lowest-order term in this expansion is the GP result (2) first derived by Lenz [34]. However, expression (6), although it gives the leading correction for larger $a n^{1 / 3}$, diverges in the strong-coupling regime, and hence has only limited validity along the BEC-unitarity crossover.

In addition to studying the system in the weak-coupling limit (2) and unitarity (4), we also consider the system along the full BEC-unitarity crossover from weak to strong coupling, as the parameter $a n^{1 / 3}$ is increased. For this purpose we consider the following minimal crossover model for the bulk chemical potential consistent with weak and strong couplings $[9,16]$ :

$$
\begin{gathered}
\bar{\mu}(a, N)=4 \pi n^{2 / 3} f(\chi), \quad \chi=a n^{1 / 3}, \\
f(\chi)=\left[\frac{\chi+(1+v) \alpha \chi^{5 / 2}}{1+v \alpha \chi^{3 / 2}+(1+v) \gamma \chi^{5 / 2}}\right],
\end{gathered}
$$

where $\alpha=32 /(3 \sqrt{\pi}), \alpha / \gamma=\xi\left(6 \pi^{2}\right)^{2 / 3} / 8 \pi$, and $\nu$ is the only free parameter in this expression. The parameters $\alpha$ and $\gamma$ are determined by the constraints that expression (7) be consistent with the LHY correction (6) as well as the unitarity limit (4), both independent of the parameter $\xi$. Expression (7) is weakly sensitive to $v$, and a smooth interpolation between the weak and strong-coupling regimes is obtained for any small $\nu$. In this study we use $v=1$. This value of $v$ was used [9] successfully in a study of ${ }^{6} \mathrm{Li}_{2} \mathrm{BEC}$ in the BEC-unitarity crossover. Expression (7) can also reproduce fairly well [30] the energies of diffusion Monte Carlo (DMC) calculation [35] of a trapped bosonic system of a small number of atoms. It was found that the energies obtained from the crossover model (7) for that bosonic system are in better agreement with the DMC calculation than those obtained from the GP equation.

The crossover model (7) is Galilei invariant and yields the hydrodynamic equations of the dipolar BEC at zero temperature, and it enables one to study collective dynamical properties of the system in the full crossover from weakcoupling to unitarity [16,30]. Equations (1) and (7) should be considered as a generalization of the GP equation with beyond mean-field corrections to properly include the effect of interaction for large positive scattering length. The saturation of the interaction at unitarity is properly taken care of in the crossover model (7). As an application we shall study here the properties of a disk-shaped dipolar BEC and dipolar BEC vortex in the strong-coupling regime to show the sensitivity of the result to the universal parameter $\xi$.

\section{Variational approximation at unitarity}

At unitarity, the dipolar mean-field equations (1), (3), and (4) can be conveniently solved by a time-dependent Lagrangian variational approach. This can be used to study the size and frequencies of oscillation of the dipolar BEC at unitarity. This is done by reducing Eq. (1) to a system of second-order nonlinear ordinary differential equations 
involving the variational parameters. The Lagrangian density of Eq. (1) is given by [28]

$$
\begin{aligned}
\mathcal{L}= & \frac{i}{2}\left(\phi \phi_{t}^{\star}-\phi^{\star} \phi_{t}\right)+\frac{1}{2}|\nabla \phi|^{2}+\frac{1}{2}\left(\rho^{2}+\lambda^{2} z^{2}\right)|\phi|^{2} \\
& +\frac{3 \xi}{10}\left(6 \pi^{2} N\right)^{2 / 3}|\phi|^{10 / 3}+\frac{N}{2}|\phi|^{2} \int U_{d d}\left(\mathbf{r}-\mathbf{r}^{\prime}\right)\left|\phi\left(\mathbf{r}^{\prime}\right)\right|^{2} d \mathbf{r}^{\prime}
\end{aligned}
$$

Recalling that $n=N|\phi|^{2}$, it can be straightforwardly verified that Eqs. (1) and (4) are the Euler-Lagrange equations for the Lagrangian density (9) [36], which should be used in the variational formulation [37]. To develop the variational approximation, we consider the following Gaussian ansatz for the wave function [28]:

$$
\phi(\mathbf{r}, t)=\sqrt{\frac{\pi^{-\frac{3}{2}}}{w_{\rho}^{2} w_{z}}} \exp \left[-\frac{\rho^{2}}{2 w_{\rho}^{2}}-\frac{z^{2}}{2 w_{z}^{2}}+i \alpha \rho^{2}+i \delta z^{2}\right],
$$

where the time-dependent variational parameters $w_{\rho}$ and $w_{z}$ are the radial and axial widths and $\alpha$ and $\delta$ are the chirps. The Lagrangian density can be calculated by substituting the wave function (10) in Eq. (9). Then the effective Lagrangian $L \equiv \int \mathcal{L} d \mathbf{r}$ becomes

$$
\begin{aligned}
L= & \frac{1}{2}\left(2 w_{\rho}^{2} \dot{\alpha}+w_{z}^{2} \dot{\delta}\right)+\frac{1}{2}\left(\frac{1}{w_{\rho}^{2}}+\frac{1}{2 w_{z}^{2}}+4 w_{\rho}^{2} \alpha^{2}+2 w_{z}^{2} \delta^{2}\right) \\
& +\frac{1}{4}\left(2 w_{\rho}^{2}+\lambda^{2} w_{z}^{2}\right)-\frac{N a_{d d}}{\sqrt{2 \pi}} \frac{f(\kappa)}{w_{\rho}^{2} w_{z}}+\frac{9 \mathcal{C}}{w_{z}^{2 / 3} w_{\rho}^{4 / 3}},
\end{aligned}
$$

where $\mathcal{C}=\sqrt{3} \xi\left(6 \pi^{2} N\right)^{2 / 3} /(50 \pi \sqrt{5}), \kappa=w_{\rho} / w_{z}$, and

$$
f(\kappa)=\frac{1+2 \kappa^{2}}{1-\kappa^{2}}-\frac{3 \kappa^{2} \tanh ^{-1} \sqrt{1-\kappa^{2}}}{\left(1-\kappa^{2}\right)^{\frac{3}{2}}} .
$$

The corresponding Euler-Lagrange equations governing the evolution of the widths $w_{\rho}$ and $w_{z}$ yield

$$
\begin{gathered}
\ddot{w}_{\rho}+w_{\rho}=\frac{1}{w_{\rho}^{3}}-\frac{a_{d d}}{\sqrt{2 \pi}} \frac{N g(\kappa)}{w_{\rho}^{3} w_{z}}+\frac{12 \mathcal{C}}{w_{z}^{2 / 3} w_{\rho}^{7 / 3}}, \\
\ddot{w}_{z}+\lambda^{2} w_{z}=\frac{1}{w_{z}^{3}}-\frac{a_{d d}}{\sqrt{2 \pi}} \frac{2 N}{w_{\rho}^{2} w_{z}^{2}} h(\kappa)+\frac{12 \mathcal{C}}{w_{z}^{5 / 3} w_{\rho}^{4 / 3}},
\end{gathered}
$$

where

$$
\begin{aligned}
& g(\kappa)=\frac{2-7 \kappa^{2}-4 \kappa^{4}}{\left(1-\kappa^{2}\right)^{2}}+\frac{9 \kappa^{4} \tanh ^{-1} \sqrt{1-\kappa^{2}}}{\left(1-\kappa^{2}\right)^{\frac{5}{2}}}, \\
& h(\kappa)=\frac{1+10 \kappa^{2}-2 \kappa^{4}}{\left(1-\kappa^{2}\right)^{2}}-\frac{9 \kappa^{2} \tanh ^{-1} \sqrt{1-\kappa^{2}}}{\left(1-\kappa^{2}\right)^{\frac{5}{2}}} .
\end{aligned}
$$

Equations (13a) and (13b) provide the dynamics of the evolution of radial and axial widths, respectively. One can obtain the expression for the frequencies and lowest-lying modes from these equations [38]. The widths for a stationary state can be obtained by setting $\ddot{w}_{\rho}=0$ and $\ddot{w}_{z}=0$ in
Eqs. (13a) and (13b). The chemical potential $\mu$ for the stationary state is given by

$$
\begin{aligned}
\mu= & \frac{1}{2}\left(\frac{1}{w_{\rho}^{2}}+\frac{1}{2 w_{z}^{2}}\right)+\frac{1}{4}\left(2 w_{\rho}^{2}+\lambda^{2} w_{z}^{2}\right) \\
& -2 \frac{N a_{d d}}{\sqrt{2 \pi}} \frac{f(\kappa)}{w_{\rho}^{2} w_{z}}+\frac{5}{3} \frac{9 \mathcal{C}}{w_{z}^{2 / 3} w_{\rho}^{4 / 3}} .
\end{aligned}
$$

\section{NUMERICAL CALCULATION}

We perform numerical simulation of the three-dimensional (3D) GP equation (1) using the split-step Crank-Nicolson method [39]. The evaluation of the dipolar integral term in this equation in coordinate space is not straightforward due to the divergence at short distances. However, this has been tackled by evaluating the dipolar term in the momentum $(\mathrm{k})$ space. The integral can be simplified in Fourier space by means of convolution as [25]

$$
\int d \mathbf{r}^{\prime} U_{d d}\left(\mathbf{r}-\mathbf{r}^{\prime}\right)\left|\phi\left(\mathbf{r}^{\prime}\right)\right|^{2}=\mathcal{F}^{-1}\left\{\mathcal{F}\left[U_{d d}\right](\mathbf{k}) \mathcal{F}\left[|\phi|^{2}\right](\mathbf{k})\right\}(\mathbf{r}),
$$

where $\mathcal{F}\left[\right.$ ] and $\mathcal{F}^{-1}\{\}$ are the Fourier transform (FT) and inverse FT, respectively. The FT of the dipole potential is known analytically [25]. The FT of density $|\phi|^{2}$ is evaluated numerically by means of a standard fast FT (FFT) algorithm. The dipolar integral in Eq. (1) involving the FT of density multiplied by FT of dipolar interaction is evaluated by the convolution theorem (16). The inverse FT is taken by means of a standard FFT algorithm. The FFT algorithm is carried out in Cartesian coordinates, and hence the GP equation is solved in three dimensions irrespective of the symmetry of the trapping potential. In the Crank-Nicolson algorithm we used space step 0.1 and time step 0.002 and employed up to 512 space discretization points in each Cartesian direction. We made an error analysis of the results for chemical potential and rms sizes and found that the maximum numerical error of the results reported here is less than $0.5 \%$.

\section{A. Experimental considerations}

Of the experimental dipolar BECs, ${ }^{52} \mathrm{Cr}$ and ${ }^{164} \mathrm{Dy}$, realized so far, the magnetic moment of ${ }^{52} \mathrm{Cr}$ is $\tilde{\mu}=6 \mu_{B}$ [19], where $\mu_{B}$ is the Bohr magneton, and that of ${ }^{164} \mathrm{Dy}$ is $\tilde{\mu}=10 \mu_{B}$ [22]. Consequently $a_{d d} \equiv \mu_{0} \tilde{\mu}^{2} m /\left(12 \pi \hbar^{2}\right)=15 a_{0}$ for ${ }^{52} \mathrm{Cr}$ and $a_{d d}=130 a_{0}$ for ${ }^{164} \mathrm{Dy}$, with $a_{0}$ the Bohr radius. Hence the dipolar interaction in ${ }^{164} \mathrm{Dy}$ is about 9 times stronger than in ${ }^{52} \mathrm{Cr}$, and we employ a ${ }^{164} \mathrm{Dy}$ BEC in this study. For ${ }^{164}$ Dy an estimate for the scattering length is $a \approx 100 a_{0}$ [22]. In the actual experiment on ${ }^{164}$ Dy a dipolar BEC of 15000 atoms in a fully anisotropic trap with frequencies $\left\{f_{x}, f_{y}, f_{z}\right\}=\{380,500,1570\} \mathrm{Hz}$ was obtained [22]. In this study, to simulate this experiment [22], we use the frequencies $\left\{f_{x}, f_{y}, f_{z}\right\}=\{436,436,1570\} \mathrm{Hz}$, so that $\lambda=3.601$, where we take a geometrical mean of the frequencies in $x$ and $y$ directions to generate an axially symmetric BEC. The length scale employed here, for $\omega=2 \pi \times 436 \mathrm{~Hz}$, is $l_{0}=$ $\sqrt{\hbar /(m \omega)}=0.376 \mu \mathrm{m}$.

Next we summarize the different theoretical and experimental estimates of $\xi$ obtained so far for bosons and fermions. 
TABLE I. Theoretical result and experimental evaluation of the parameter $\beta \equiv(\xi-1)$ for the Bose and Fermi gases.

\begin{tabular}{|c|c|c|}
\hline Fermi, theory & $\begin{array}{r}\text { Astrakharchik et al. [3] } \\
\text { Carlson et al. }[4] \\
\text { Perali } \text { et al. }[40]\end{array}$ & $\begin{array}{l}-0.58 \\
-0.58 \\
-0.545\end{array}$ \\
\hline Fermi, expt $\left({ }^{6} \mathrm{Li}\right)$ & $\begin{array}{r}\text { Partridge et al. [5] } \\
\text { Kinast et al. [6] } \\
\text { Bartenstein et al. [7] } \\
\text { Navon } \text { et al. [10] } \\
\text { Luo and Thomas [8] }\end{array}$ & $\begin{array}{c}-0.54(5) \\
-0.49(4) \\
-0.73_{-0.09}^{+0.12} \\
-0.59(1) \\
\approx-0.6\end{array}$ \\
\hline Fermi, expt $\left({ }^{40} \mathrm{~K}\right)$ & Stewart et al. [11] & $-0.54_{-0.12}^{+0.05}$ \\
\hline Bose, theory & $\begin{array}{r}\text { Diederix et al. [2] } \\
\text { Lee et al. [15] } \\
\text { Cowell et al. [13] } \\
\text { Song and Zhou [14] }\end{array}$ & $\begin{array}{l}-0.54 \\
-0.34 \\
<1.93 \\
<-0.2\end{array}$ \\
\hline Analysis, expt $\left({ }^{6} \mathrm{Li}_{2}\right)$ [7] & Adhikari [9] & $<0.6$ \\
\hline Bose, expt $\left({ }^{7} \mathrm{Li}\right)$ & Navon et al. [12] & $>-0.56(8)$ \\
\hline
\end{tabular}

Often the parameter $\xi$ is written as $\xi \equiv(1+\beta)$, and different estimates of $\beta$ are given in Table I, where the variational calculations of Refs. [13,15] for bosons are upper bounds, and the experimental result of Ref. [12] for ${ }^{7} \mathrm{Li}$ is a lower bound only. Yet another estimate of $\xi$ can be obtained from a consideration of Fermi superfluid in the BEC side of the Bardeen-Cooper-Schrieffer-BEC (BCS-BEC) crossover. Here we reconsider an analysis [9] of the experiment [7] on ${ }^{6} \mathrm{Li}$ in the BEC side of BEC-unitarity crossover. The molecular BEC of ${ }^{6} \mathrm{Li}_{2}$ was then studied using Eqs. (1), (7), and (8) but with $\alpha / \gamma=\xi_{\text {mol }}\left(6 \pi^{2}\right)^{2 / 3} /(2 \pi)$, where $\xi_{\text {mol }}$ is the universal parameter of Ref. [9], in place of $\alpha / \gamma=\xi\left(6 \pi^{2}\right)^{2 / 3} /(8 \pi)$ considered here. This implies that for a comparison of the two studies we should take $\xi=4 \xi_{\text {mol }}$. The analysis of Ref. [9] yielded $\xi_{\text {mol }} \approx 0.4$, so that $\xi \approx 1.6$ corresponding to $\beta \approx 0.6$. In that analysis [9] it was assumed that the bosonic molecular unitarity of ${ }^{6} \mathrm{Li}_{2}$ was achieved for the same strength of atomic interaction as the fermionic unitarity of ${ }^{6} \mathrm{Li}$. Actually the bosonic molecular unitarity should be achieved at a different value of interaction and into the BEC side of the BCS-BEC crossover, where the system is less repulsive. This would lead to a smaller value of the parameter $\xi(\xi<1.6)$ and $\beta$. Hence the analysis of Ref. [9] gives an upper bound. From the results reported in Table I, the most accurate theoretical [3,4] and experimental $[8,10]$ estimates for a Fermi gas converge to a value of $\xi$ very close to 0.4 .

\section{B. Disk-shaped dipolar Bose-Einstein condensate}

We study a disk-shaped dipolar BEC of $15000{ }^{164}$ Dy atoms with $a_{d d}=130 a_{0}$ as in the experiment of Lu et al. [22]. The parameter $\xi$ can be extracted from the observables of the dipolar BEC in the strong-coupling regime where the observables would be sensitive to this parameter. For this purpose, in this paper, in addition to the numerical study at unitarity, we also present a complete numerical study of the dipolar BEC in the strong-coupling regime for scattering length $a>100 a_{0}$ using the crossover model (7).

In Fig. 1(a) we plot the radial density of the BEC along $x$ axis $|\Phi(x, y=0)|^{2}$ obtained by integrating out the $z$
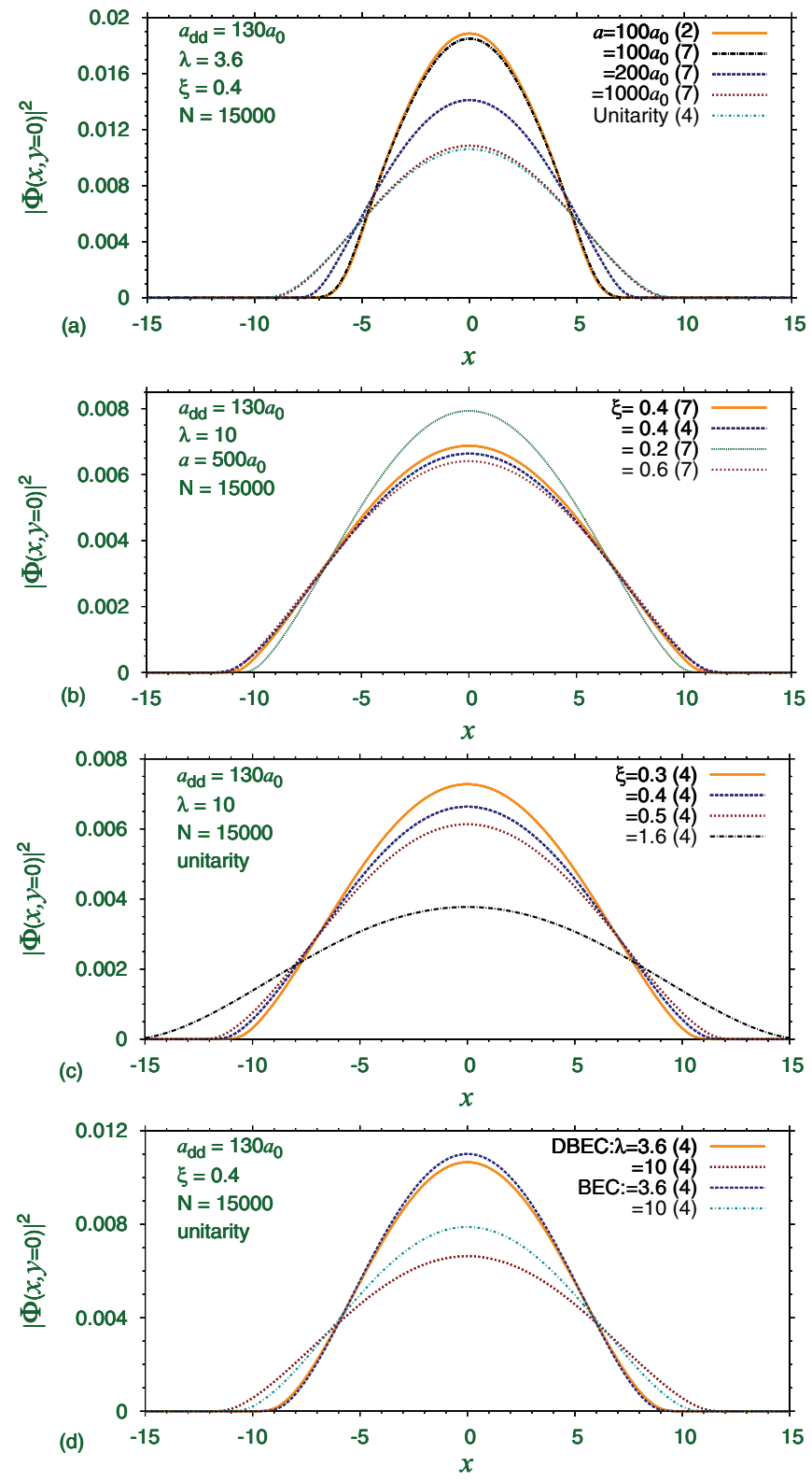

FIG. 1. (Color online) (a) Radial density along $x$ axis $\mid \Phi(x, y=$ $0)\left.\right|^{2}=\int d z|\phi(x, y=0, z)|^{2}$ of a ${ }^{164}$ Dy BEC with $N=15000, \lambda=$ $3.6, a_{d d}=130 a_{0}, v=1$, and $\xi=0.4$ for $a=100 a_{0}, 200 a_{0}, 1000 a_{0}$, and at unitarity using the GP limit (2), crossover model (7), and at unitarity (4). (b) The same for $\lambda=10, a=500 a_{0}$ and $\xi=0.2,0.4,0.6$ using the crossover model (7). The density at unitarity (4) for $\xi=0.4$ is also shown. (c) The same for $\lambda=10$ and different $\xi$ at unitarity (4). (d) The same for $\xi=0.4, \lambda=3.6,10$ at unitarity (4) for $a_{d d}=130 a_{0}$ (DBEC) and $a_{d d}=0$ (BEC).

dependence of density: $|\Phi(x, y)|^{2}=\int d z|\phi(x, y, z)|^{2}$. In this figure we show the result for $a=100 a_{0}$ using the GP model (2) and at unitarity (4) in addition to the results for $a=$ $100 a_{0}, 300 a_{0}$, and $500 a_{0}$ using the BEC-unitarity crossover model (7) with $\xi=0.4$ and $v=1$. For small $a$, the density from the crossover model (7) is in agreement with the GP model (2) and hence practically independent of the parameter $\xi$, whereas for large $a$ it approximates the unitarity limit (4) with the increase of $a$. In Fig. 1(b) we plot the radial density for $a=500 a_{0}$ for different $\xi$ using the crossover model (7). 

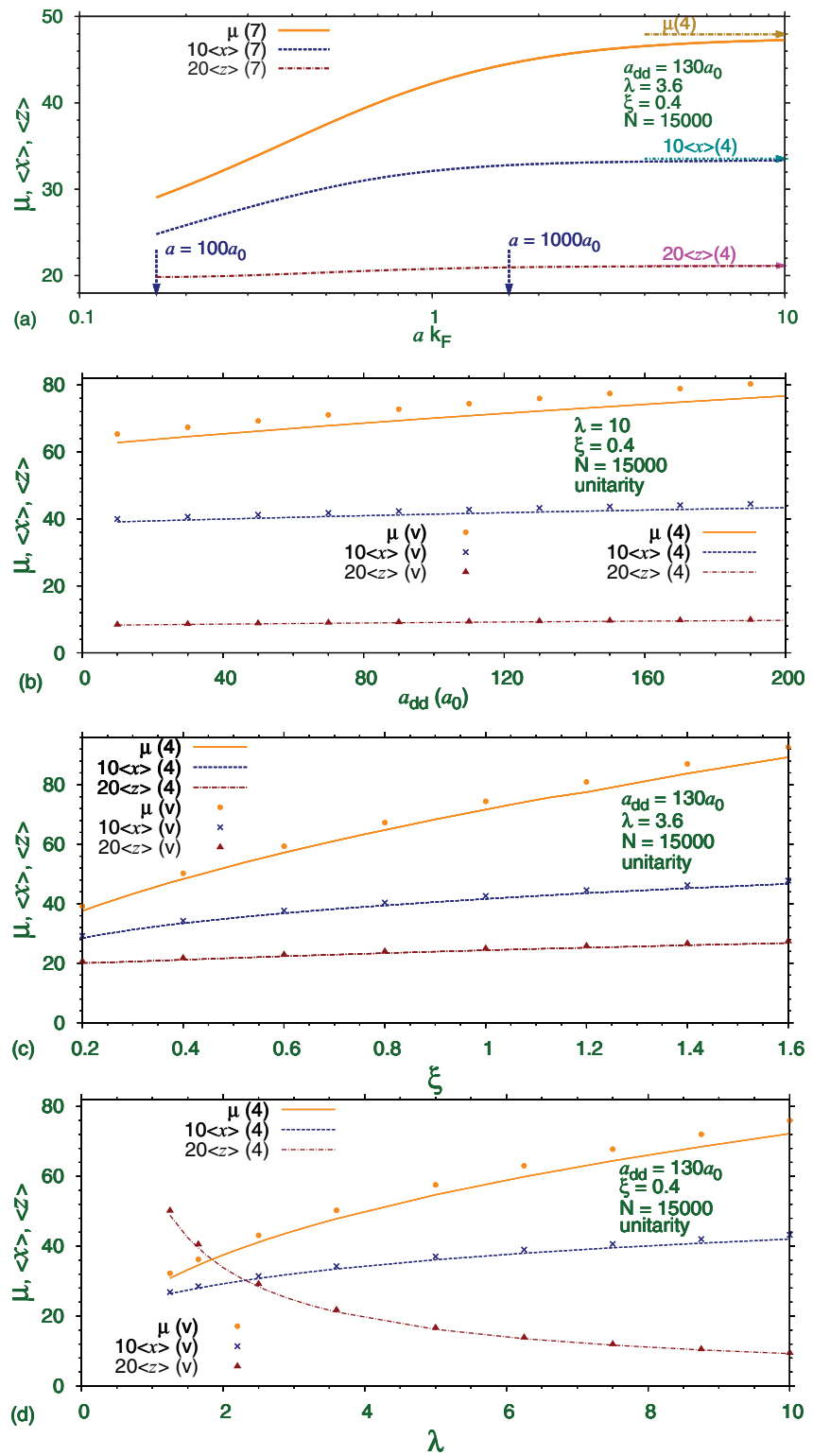

FIG. 2. (Color online) (a) The numerical rms sizes $\langle x\rangle,\langle z\rangle$, and chemical potential $\mu$ of the dipolar BEC with $N=15000, a_{d d}=$ $130 a_{0}, \xi=0.4, v=1, \lambda=3.6$ vs $a k_{F}$ using the crossover model (7), as well as at unitarity (4) (arrow). (b) The numerical (4) and variational (v) results for the same at unitarity versus $a_{d d}$. (c) The numerical (4) and variational (v) results for the same at unitarity versus $\xi$. (d) The numerical (4) and variational (v) results for the same at unitarity vs $\lambda$.

The result at unitarity (4) for $\xi=0.4$ is also shown. The density is sensitive to the parameter $\xi$ for $a=500 a_{0}$ as can be seen from Fig. 1(b) comparing the results for $\xi=0.2,0.4$, and 0.6 . The sensitivity of the density on $\xi$ at unitarity is illustrated in Fig. 1(c), where we show the radial density for $\xi=0.3,0.4,0.5$, and 1.6. Finally, in Fig. 1(d) we show the density at unitarity for nondipolar and dipolar BECs for two values of the trap asymmetry $\lambda=3.6$ and 10 . The difference between the two densities is more pronounced for $\lambda=10$, where the dipolar repulsion is stronger.

In Fig. 2(a) we plot the rms sizes of the BEC $\langle x\rangle,\langle z\rangle$, and the chemical potential $\mu$ for $\xi=0.4$, and $a_{d d}=130 a_{0}$ as calcu- lated using crossover model (7), versus the dimensionless parameter $k_{F} a$, where $k_{F}$ is the Fermi wave vector in a harmonic trap defined by $k_{F}=(48 N)^{1 / 6} / \bar{l}[1]$, where $\bar{l}=\sqrt{\hbar / m \bar{\omega}}, \bar{\omega}=$ $2 \pi\left(f_{x} f_{y} f_{z}\right)^{1 / 3},\left\{f_{x}, f_{y}, f_{z}\right\}=\{436,436,1570\}$ Hz. One can find from Fig. 2(a) how these three quantities, $\langle x\rangle,\langle z\rangle$, and $\mu$, approach their values at unitarity as $a$ increases. With the increase of $a$ these quantities saturate rapidly to their respective values at unitarity. In Fig. 2(b), we plot the numerical (n) and variational (v) results for $\langle x\rangle,\langle z\rangle$, and $\mu$ versus $a_{d d}$ at unitarity, which shows that the results are weakly sensitive to a variation of $a_{d d}$. In Fig. 2(c) we plot $\langle x\rangle,\langle z\rangle$, and $\mu$ versus $\xi$ at unitarity, which shows that the results are sensitive to a variation of $\xi$. Finally, in Fig. 2(d) we plot $\langle x\rangle,\langle z\rangle$, and $\mu$ versus $\lambda$ at unitarity showing the strong sensitivity of the results to a variation of $\lambda$. From Figs. 2(b), 2(c), and 2(d) we see that the variational results are in good agreement with the numerical ones.

In a recent experiment, Navon et al. [12] were able to make measurements for densities of a very dilute BEC of ${ }^{7} \mathrm{Li}$ for $a=2150 a_{0}$ and extract the parameter $\xi$ from a theoretical analysis using the LHY correction $[31,33]$. The very dilute BEC prepared in a weak trap allowed one to make an experiment for large $a=2150 a_{0}$. But because of the low density, the BEC remained away from the strong-coupling regime even for $a=2150 a_{0}$ and was studied by the LHY correction, rather than a full crossover model as in the present study. In this regime the densities are weakly sensitive to the parameter $\xi$, and only an upper limit $\xi<0.6$ could be obtained from that study [12].

Apart from density profile and rms sizes of the dipolar BEC, other observables that can be studied are the frequencies of radial and axial oscillations $\Omega_{\rho}$ and $\Omega_{z}$, respectively, of the fundamental modes. We calculated these frequencies by numerically solving the variational equations (13a) and (13b)
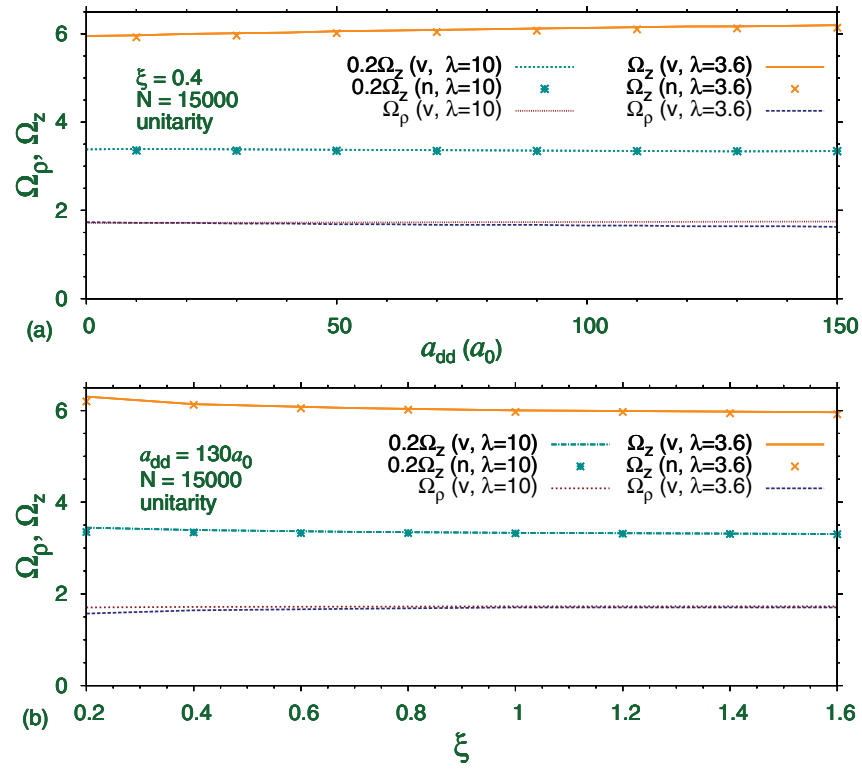

FIG. 3. (Color online) (a) Variational frequency (v) of radial and axial oscillation $\Omega_{\rho}$ and $\Omega_{z}$ of the dipolar BEC with $N=15000, \xi=$ $0.4, v=1, \lambda=3.6,10$ vs $a_{d d}$ at unitarity from Eqs. (13a) and (13b). The numerical frequencies (n) of axial oscillation obtained from a solution of Eqs. (1) and (7) are also shown. (b) The same for $a_{d d}=$ $130 a_{0}$ vs $\xi$. 
in different cases. The initial widths $w_{\rho}$ and $w_{z}$ were taken as their equilibrium static values, and their time evolution is obtained. The frequencies of radial and axial oscillations were extracted from the time evolution of the respective widths. In Fig. 3(a) we show these frequencies for $\xi=0.4$ versus $a_{d d}$, and in Fig. 3(b) we plot these frequencies for $a_{d d}=130 a_{0}$ versus $\xi$ for $\lambda=3.6,10$. We also calculated the axial frequency $\Omega_{z}$ from the small oscillation of the rms axial size of the BEC upon realtime evolution of the mean-field equations (1) and (4). Because of a mixture of frequencies of higher modes, it was not possible to obtain precisely the frequency $\Omega_{\rho}$ from a solution of the mean-field equations. The mean-field and the variational results for $\Omega_{z}$ are in good agreement with each other. These frequencies are practically insensitive to a variation of $a_{d d}$ as well as of $\xi$. Hence it may not be very fruitful to study these frequencies in the strong-coupling regime in order to extract the parameter $\xi$, especially for a moderate density as in this study.

\section{Dipolar BEC vortex}

Next we study the density of a disk-shaped dipolar BEC vortex of unit angular momentum for strong coupling and demonstrate the sensitivity of the result on the parameter $\xi$. In this case the radius of the vortex core is an observable directly related to the healing length [1] of the BEC and will also be considered. The radius of the vortex core $\rho_{c}$ is defined as the radial distance from the center of the vortex to a point where the density increases to the maximum value. It is more appropriate to consider the relative radius of vortex core defined by $r_{0} \equiv$ $\rho_{c} /\langle x\rangle$, which gives the vortex core radius in relation to the radial size of the condensate. It is demonstrated that the relative vortex core radius $r_{0}$ could be sensitive to $\xi$ in the strongcoupling regime and could be useful in deciding the value of $\xi$.
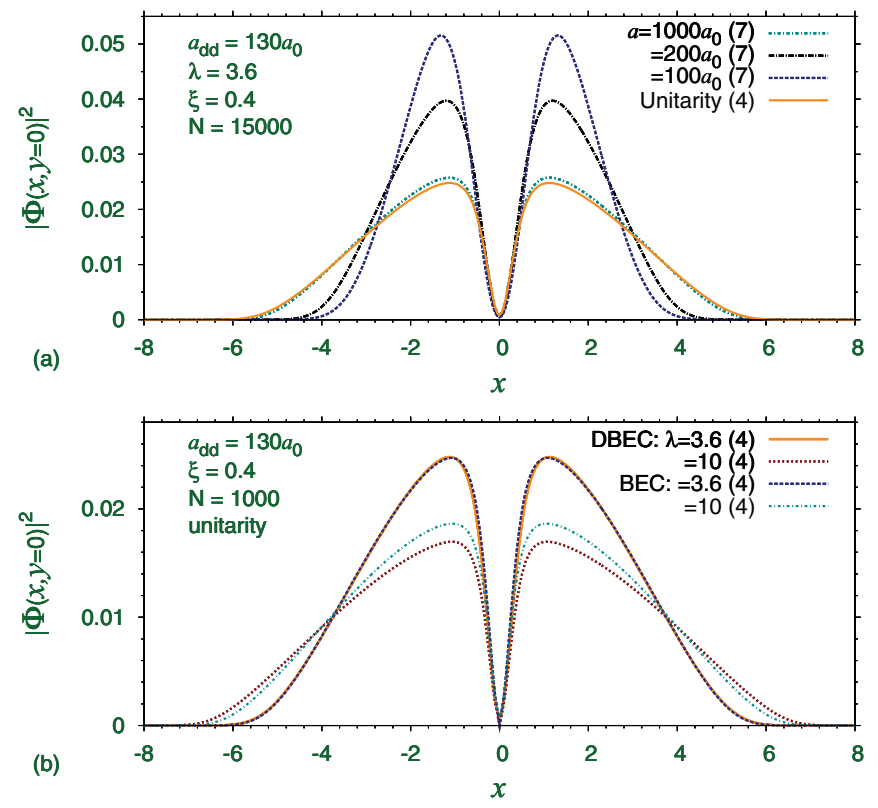

FIG. 4. (Color online) (a) Radial density along $x$ axis $\mid \Phi(x, y=$ $0)\left.\right|^{2}$ of a ${ }^{164}$ Dy BEC vortex of $N=1000, \lambda=3.6, a_{d d}=130 a_{0}, v=$ 1 , and $\xi=0.4$ for $a=100 a_{0}, 200 a_{0}, 1000 a_{0}$, and at unitarity using the crossover model (7), and at unitarity (4). (b) The same for $\xi=$ $0.4, \lambda=3.6,10$ at unitarity (4) for $a_{d d}=130 a_{0}(\mathrm{DBEC})$, and $a_{d d}=0$ (BEC).
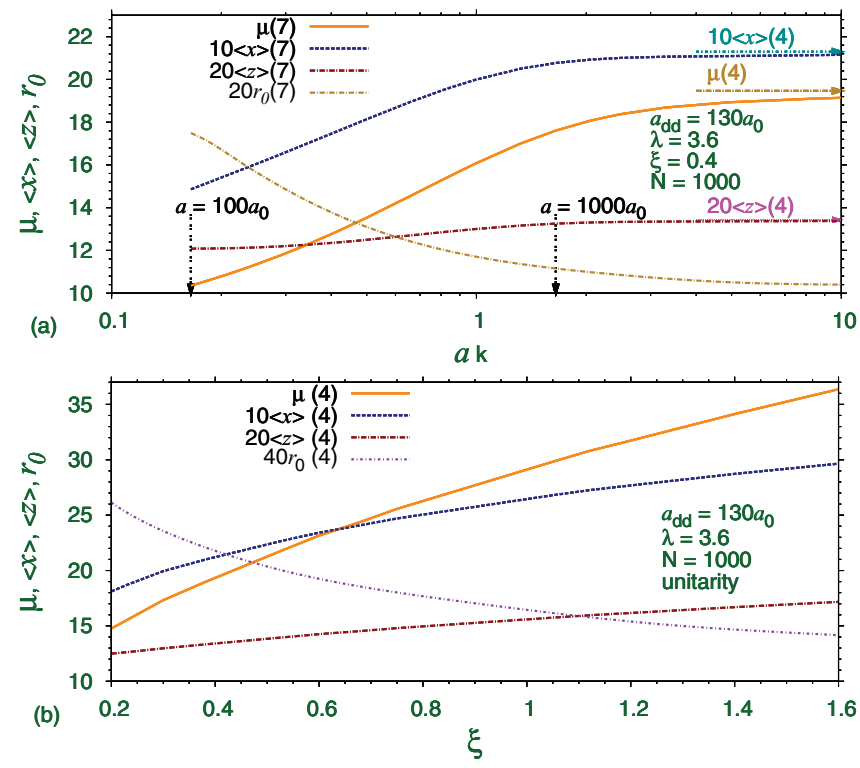

FIG. 5. (Color online) (a) The rms sizes $\langle x\rangle,\langle z\rangle$, chemical potential $\mu$, and relative radius of vortex core $r_{0}$ of the dipolar BEC vortex with $N=1000, a_{d d}=130 a_{0}, \xi=0.4, v=1, \lambda=3.6$ vs $a k_{F}$ using the crossover model (7), as well as at unitarity (4) (arrow). (b) The same at unitarity (4) vs $\xi$.

In Fig. 4(a) we plot the radial density of the BEC vortex along the $x$ axis $|\Phi(x, y=0)|^{2}$ for $N=1000, \lambda=3.6, a_{d d}=$ $130 a_{0}, \xi=0.4, v=1$ for different values of scattering length $a$. In this figure we show the result for $a=100 a_{0}$ and at unitarity (4) in addition to the results for $a=100 a_{0}, 200 a_{0}$, and $1000 a_{0}$ using the BEC-unitarity crossover model (7). For small $a$, the density obtained using the crossover model (7) is in agreement with the GP equation (2) and hence independent of the parameter $\xi$, whereas for large $a$ it approximates the unitarity limit (4). In Fig. 4(b) we show the radial density at unitarity for nondipolar and dipolar BECs for two values of the trap asymmetry $\lambda=3.6$ and 10 . The difference between the two densities is more pronounced for $\lambda=10$, where the dipolar repulsion is stronger.

In Fig. 5(a) we plot chemical potential $\mu$ and rms sizes $\langle x\rangle$, $\langle z\rangle$ together with the relative radius of vortex core $r_{0}$ versus $a k_{F}$ for $N=1000, a_{d d}=130 a_{0}, \lambda=3.6, \xi=0.4, v=1$ obtained using the crossover model (7). The result at unitarity (4) is also shown. The relative radius of vortex core reduces with the increase of the scattering length. Similar reduction of the radius of vortex core was predicted for a nondipolar BEC before [16,41]. In Fig. 5(b) we plot $\mu,\langle x\rangle,\langle z\rangle, r_{0}$ versus $\xi$ at unitarity (4) for $N=1000, \lambda=3.6, a_{d d}=130 a_{0}, \lambda=3.6, v=1$. As scattering length $a$ increases in Fig. 5(a) or the parameter $\xi$ increases in Fig. 5(b), the system becomes more repulsive leading to a smaller healing length. Consequently the relative radius of vortex core $r_{0}$, which is closely related to the healing length, decreases [1]. The relative radius of vortex core shows much sensitivity to the scattering length $a$ and $\xi$.

\section{CONCLUSION}

The properties of a BEC at unitarity is controlled by a universal parameter $\xi$ relating the energies of noninteracting 
and unitary uniform gases. Using the BEC-unitarity crossover model (7) we studied the properties of a disk-shaped dipolar BEC and dipolar BEC vortex in the strong-coupling regime. We find that the density profiles are sensitive to the parameter $\xi$ in this regime, and a study of density should yield an information about this parameter. We also studied the frequencies of the fundamental modes of radial and axial oscillation of this BEC and find that they are not much sensitive to $\xi$. For a dipolar $\mathrm{BEC}$ vortex, in addition to density, the relative radius of vortex core is also found to be sensitive to $\xi$ in the strong-coupling regime, so that a study of this radius may reveal information about $\xi$. Also to extract the parameter $\xi$ it is not necessary to study the system at unitarity. The density profile of the BEC is sensitive to the parameter $\xi$ for the contact interaction lying between the weak-coupling GP and strong-coupling unitarity limits, so that a study in this regime should reveal information about this parameter. In this study we used a dipolar BEC of $15000{ }^{164}$ Dy atoms in a disk-shaped trap of anisotropy $\lambda=3.6$, as in the experiment of Ref. [22], and $\lambda=10$. For an experimental study the anisotropy of $\lambda=10$, or larger, and a $\mathrm{BEC}$ with strong dipole interaction is to be preferred.

\section{ACKNOWLEDGMENT}

We thank FAPESP (Brazil), CNPq (Brazil), DST (India), and CSIR (India) for partial support.
[1] S. Giorgini, L. Pitaevskii, and S. Stringari, Rev. Mod. Phys. 80, 1215 (2008).

[2] J. M. Diederix, T. C. F. van Heijst, and H. T. C. Stoof, Phys. Rev. A 84, 033618 (2011).

[3] G. E. Astrakharchik, J. Boronat, J. Casulleras, and S. Giorgini, Phys. Rev. Lett. 93, 200404 (2004).

[4] J. Carlson and S. Reddy, Phys. Rev. Lett. 95, 060401 (2005); J. Carlson, S. Y. Chang, V. R. Pandharipande, and K. E. Schmidt, ibid. 91, 050401 (2003)

[5] G. Partridge et al., Science 311, 503 (2006).

[6] J. Kinast, A. Turlapov, and J. E. Thomas, Phys. Rev. Lett. 94, 170404 (2005).

[7] M. Bartenstein, A. Altmeyer, S. Riedl, S. Jochim, C. Chin, J. H. Denschlag, and R. Grimm, Phys. Rev. Lett. 92, 120401 (2004).

[8] L. Luo and J. E. Thomas, J. Low Temp. Phys. 154, 1 (2009).

[9] S. K. Adhikari, J. Phys. B 43, 085304 (2010).

[10] N. Navon, S. Nascimbène, F. Chevy, and C. Salomon, Science 328, 729 (2010).

[11] J. T. Stewart, J. P. Gaebler, C. A. Regal, and D. S. Jin, Phys. Rev. Lett. 97, 220406 (2006)

[12] N. Navon, S. Piatecki, K. Gunter, B. Rem, T. C. Nguyen, F. Chevy, W. Krauth, and C. Salomon, Phys. Rev. Lett. 107, 135301 (2011).

[13] S. Cowell, H. Heiselberg, I. E. Mazets, J. Morales, V. R. Pandharipande, and C. J. Pethick, Phys. Rev. Lett. 88, 210403 (2002).

[14] J. L. Song and F. Zhou, Phys. Rev. Lett. 103, 025302 (2009).

[15] Y.-L. Lee and Y.-W. Lee, Phys. Rev. A 81, 063613 (2010).

[16] S. K. Adhikari and L. Salasnich, Phys. Rev. A 77, 033618 (2008).

[17] S. B. Papp, J. M. Pino, R. J. Wild, S. Ronen, C. E. Wieman, D. S. Jin, and E. A. Cornell, Phys. Rev. Lett. 101, 135301 (2008).

[18] S. E. Pollack, D. Dries, M. Junker, Y. P. Chen, T. A. Corcovilos, and R. G. Hulet, Phys. Rev. Lett. 102, 090402 (2009).

[19] T. Koch, T. Lahaye, J. Metz, B. Frohlich, A. Griesmaier, and T. Pfau, Nat. Phys. 4, 218 (2008).

[20] T. Lahaye et al., Nature (London) 448, 672 (2007); T. Lahaye, C. Menotti, L. Santos, M. Lewenstein, and T. Pfau, Rep. Prog. Phys. 72, 126401 (2009).

[21] M. Lu, S. H. Youn, and B. L. Lev, Phys. Rev. Lett. 104, 063001 (2010); J. J. McClelland and J. L. Hanssen, ibid. 96, 143005 (2006); S. H. Youn, M. W. Lu, U. Ray, and B. V. Lev, Phys. Rev. A 82, 043425 (2010).
[22] M. Lu, N. Q. Burdick, Seo Ho Youn, and B. L. Lev, Phys. Rev. Lett. 107, 190401 (2011).

[23] S. Inouye et al., Nature (London) 392, 151 (1998).

[24] P. Muruganandam and S. K. Adhikari, Phys. Lett. A 376, 480 (2012); T. Lahaye, J. Metz, B. Frohlich, T. Koch, M. Meister, A. Griesmaier, T. Pfau, H. Saito, Y. Kawaguchi, and M. Ueda, ibid. 101, 080401 (2008); C. Ticknor, R. M. Wilson, and J. L. Bohn, ibid. 106, 065301 (2011); C. Krumnow and A. Pelster, Phys. Rev. A 84, 021608 (2011); I. Tikhonenkov, B. A. Malomed, and A. Vardi, Phys. Rev. Lett. 100, 090406 (2008); R. Nath, P. Pedri, and L. Santos, ibid. 102, 050401 (2009).

[25] K. Góral and L. Santos, Phys. Rev. A 66, 023613 (2002).

[26] S. Ronen, D. C. E. Bortolotti, and J. L. Bohn, Phys. Rev. Lett. 98, 030406 (2007); R. M. Wilson, S. Ronen, J. L. Bohn, and H. Pu, ibid. 100, 245302 (2008); M. Asad-uz-Zaman and D. Blume, Phys. Rev. A 83, 033616 (2011); 80, 053622 (2009); H. Saito, Y. Kawaguchi, and M. Ueda, Phys. Rev. Lett. 102, 230403 (2009).

[27] M. Abad, M. Guilleumas, R. Mayol, M. Pi, and D. M. Jezek, Phys. Rev. A 81, 043619 (2010); O. Dutta and P. Meystre, ibid. 75, 053604 (2007); R. M. W. van Bijnen, A. J. Dow, D. H. J. O'Dell, N. G. Parker, and A. M. Martin, ibid. 80, 033617 (2009); R. M. W. van Bijnen, N. G. Parker, S. J. J. M. F. Kokkelmans, A. M. Martin, and D. H. J. O’Dell, ibid. 82, 033612 (2010); N. G. Parker, C. Ticknor, A. M. Martin, and D. H. J. O'Dell, ibid. 79, 013617 (2009); N. G. Parker and D. H. J. O’Dell, ibid. 78, 041601 (2008); R. M. Wilson, S. Ronen, and J. L. Bohn, ibid. 80, 023614 (2009).

[28] L. E. Young-S, P. Muruganandam, and S. K. Adhikari, J. Phys. B 44, 101001 (2011); P. Muruganandam and S. K. Adhikari, ibid. 44, 121001 (2011); S. K. Adhikari and P. Muruganandam, ibid. 45, 045301 (2012).

[29] L. Santos, G. V. Shlyapnikov, P. Zoller, and M. Lewenstein, Phys. Rev. Lett. 85, 1791 (2000).

[30] S. K. Adhikari and L. Salasnich, Phys. Rev. A 78, 043616 (2008); S. K. Adhikari, H. Lu, and H. Pu, ibid. 80, 063607 (2009).

[31] T. D. Lee, K. Huang, and C. N. Yang, Phys. Rev. 106, 1135 (1957).

[32] F. Dalfovo and S. Stringari, Phys. Rev. A 53, 2477 (1996). 
[33] A. Fabrocini and A. Polls, Phys. Rev. A 64, 063610 (2001).

[34] W. Lenz, Z. Phys. 56, 778 (1929).

[35] D. Blume and C. H. Greene, Phys. Rev. A 63, 063601 (2001).

[36] S. K. Adhikari, Phys. Rev. A 70, 043617 (2004); S. K. Adhikari and B. A. Malomed, Europhys. Lett. 79, 50003 (2007).

[37] V. M. Pérez-García, H. Michinel, J. I. Cirac, M. Lewenstein, and P. Zoller, Phys. Rev. A 56, 1424 (1997).
[38] S. Yi and L. You, Phys. Rev. A 63, 053607 (2001); Phys. Rev. Lett. 92, 193201 (2004).

[39] P. Muruganandam and S. K. Adhikari, Comput. Phys. Commun. 180, 1888 (2009).

[40] A. Perali, P. Pieri, and G. C. Strinati, Phys. Rev. Lett. 93, 100404 (2004).

[41] J.K. Nilsen, J. Mur-Petit, M. Guilleumas, M. Hjorth-Jensen, and A. Polls, Phys. Rev. A 71, 053610 (2005). 